\title{
On the Applicability of Fibre-Fed Multi-Object Spectrographs in Flare Star Research
}

\author{
Gábor Szécsényi-Nagy \\ Department of Astronomy, Eötvös Loránd University H-1083 Budapest \\ VIII. Ludovika tér 2. Hungary
}

\begin{abstract}
The use of optical fibres has recently allowed the full field of some Schmidt telescopes to be accessed for multi-object spectroscopy. The new device: the MOS (multi-object spectrograph) is to revolutionize the field of astronomical spectroscopy. Cosmic objects grouped into aggregates having apparent angular diameters of 1 to 6 degrees and similar apparent brightness in the visual or near infrared spectral range are to be the most probable future targets of MOSs. Flare stars - as it has been demonstrated convincingly by various authors (see Szecsenyi-Nagy 1986, and references therein) - are the most abundant type of variable stars but because of their low luminosity and relatively late discovery we have only very limited amount of spectroscopic data about most of these objects. It is a real bonanza that most of the catalogued flare stars are associated with young open clusters and this fact of course suggests them as really promising targets for wide-field Schmidt cameras equipped with MOSs. In an average open cluster scrutinized for its flare star members some dozen or some hundred representatives of this species are known which are distributed over a sky field of 10-20 square degrees. Fortunately, they are never packed into unresolvable concentrations and even short focal length cameras are able to produce separate images of all cluster member flare stars for an accurate photographic photometry of the objects. The apparent visual brightness of these stars most often fits a 57 magnitude range. This seems to be profitable also for the effectiveness of using fibre-fed MOSs in flare star spectroscopy. The first steps of such a program should be the study of radial velocities and the comparison of the measured values with the accepted radial velocity of the cluster. These measurements could provide new and independent arguments for (or against?) the cluster membership of the flare stars observed in the fields of catalogued open clusters. Later, more detailed spectral studies should collect information about the time dependent features of the spectra of flare stars.
\end{abstract}

\section{Introduction}

Flare stars, this new kind of variable, were discovered in the forties and were named after their most prominent representative UV Ceti. At rest these objects are very low intrinsic brightness red dwarfs. Their spectra may show emis- 
sion lines and in most cases they can be classified as dMOe...dM6e. Because their absolute visual magnitudes are between +10 and +18 only the ones in the immediate solar neighbourhood can be observed using spectroscopic methods. Unfortunately there are hardly more than 80 of that type. Some of these stars flare up often enough and offer the opportunity for recording their optical spectrum during active periods using only a reasonable amount of telescope time. (As is well known, the flare up times of any flare star are totally unpredictable but in the case of higher frequency variables one has to wait less until an event occurs). Fortunately at the beginning of the fifties it was already discovered (Haro \& Morgan 1953) that these peculiar stars seem to cluster in certain parts of the sky. Objects with similar properties to that of UV Ceti were identified in the Orion nebula and its close vicinity, though at first they were considered as a separate class of variable stars, and therefore they were called flash - not flare stars. Evidence collected during the next three decades showed that flare stars in star clusters and associations and UV Ceti stars in the solar vicinity belong very probably to the same class of variables, e.g. they are flare-active red dwarfs of various ages. All of their observed differences can be explained by age differences (Mirzoyan 1990). Flare stars have also been found in the fields of NGC 2264, NGC 7000, Coma Berenices, Hyades, Praesepe and Pleiades clusters and the TDC-1 and TOC-2 dark clouds of the Taurus constellation. From the investigation of the clusters and associations it could be concluded that these objects gather primarily in younger star aggregations, while they could be hardly found in older clusters.

\section{Statistics of Flare Stars}

A simple evaluation of the data compiled by Tsvetkov et al. 1994 demonstrates that from the 1260 acknowledged or suspected flare stars less than $8 \%$ belong to the family of solar neighbourhood stars, although up to a distance of $25 \mathrm{pc}$ these are the most abundant type of variable stars. This fact is not surprising as the majority of stars belongs to $\mathrm{dK}$ or $\mathrm{dM}$ MK-classes. More than $90 \%$ of the Hlare stars belong to fewer than ten sky fields, from which two (the Orion and the Pleiades or Eta Tauri fields) contribute at least a thousand objects. Since all discoveries were based on Schmidt plates these fields can be covered by the large field of Schmidt cameras as a rule. Only a few of the objects lay beyond the boundary of the field of view (FOV) of the telescopes.

Apparent magnitudes for the nearby flare stars range from 7.6-12.2 $(R)$ and 10.5-16.7 $(U)$ while those of the Pleiades' sources are typically 5 mag higher (this is an obvious consequence of the cluster's larger distance - the distance modulus being 5.5). It is a pity that the other system rich in flare stars, the Orion is about 4-5 times further away and is filled with interstellar matter which may cause considerable absorption in the optical band and result in additional dimming of the light of its members. These facts explain why only a very limited sample of the cluster member flare stars are classified into spectral classes and also explain the uncertainties of the classifications. Although MK classes of solar vicinity flare stars are known better than a decimal class, in the case of cluster member stars classification errors may exceed even two subclasses while no information about the presence of emission features can be given. 


\section{Methods of Multi-Object Spectroscopy}

Two traditional ways of simultaneously recording spectra of cosmic sources from a large FOV are objective grating and objective prism spectroscopy. Both have a common drawback : the sky background contributes with its total brightness to the distributed light of program stars. The result is a terribly decreased $\mathrm{S} / \mathrm{N}$ ratio and a strictly limited magnitude range. Further problems are caused by the low (less than $50 \%$ ) transmission of a grating and the considerable absorption caused by the material of a prism of reliably high refracting angle. Extra losses in limiting magnitude are caused by the widening of spectra, a technique essential for reaching higher accuracy in spectral studies. The total loss may reach 5-8.5 magnitudes depending on the resolution. The new methods of MOS are based on the use of optical fibres guiding the light of the object collected by the optical elements of the wide-field astronomical cameras into high performance spectrographs, which distribute the photons among a large number of pixels of very high quantum efficiency light detectors, normally CCDs. Now the photons arriving from the direction of the seeing disk of the program stars (i.e. light from the observed cosmic source + light from the tiny spot of the sky background corresponding to the FOV of a single fibre) are only dispersed and a gain of $100-1,000$ is easily attainable relative to the earlier methods and devices. Taking into account the higher $\mathrm{QE}$ of the silicon-based detectors fibre-fed multi-object spectrographs (FFMOSs) may provide hundreds of optical spectra of fair resolution for each flare star of a given field. Since these stars have very cool atmospheres their continuous spectra peak in the red or infrared band and this may contribute to the most effective use of CCDs which have maximum sensitivity in these spectral regions. Although the positioning of the individual fibres is a long and tedious procedure, the number of the fibres is increased until vignetting caused by the system is not too disturbing.

\section{Flare Stars as Potential Targets for FFMOSs}

FFMOSs may be used for simultaneous medium-resolution spectroscopy of faint objects which may be spread over sky fields of 2-5 degrees wide and may differ in apparent brightness by several magnitudes, depending on the dynamic range of the photon detector built into the spectrograph. The number of targets must be somewhat less than the number of fibres because sky background spectra are needed for the reduction and some reference sources for the calibration of the spectra. Consequently the existing systems are able to follow about 50-200 objects or more at once.

Catalogued flare stars seem to be very promising targets for FFMOSs. In some fields (which are practically equal to Schmidt camera fields) hundreds of faint flare stars were discovered. These are not grouped in to undetached condensations, consequently most of them are within easy reach of the optical fibres. Many of the flare stars of star cluster fields are much brighter than $V=17$ or $R=18$ and a considerable fraction of these objects may be emission line stars. We know almost nothing about their radial velocities. The proper motion of the brightest cluster member flare stars is determined and that of the fainter ones is being studied (Schilbach et al. 1994), but in order to be able to compute their 
space velocities their radial velocities have to be determined by measuring the Doppler-shift of their spectral features. These measurements could provide independent information on the membership probabilities of flare stars found in the fields of star clusters and associations. Based on spectra of medium resolution, the accuracy of the classification of these objects could be increased spectacularly and a straightforward comparison of the physical parameters characterizing the solar vicinity and more distant flare stars (which may belong to systems of various ages) could be accomplished. Furthermore the method of simultaneous observations offers definitely positive chances of capturing the spectrum of a flare phenomenon. Amongst the Eta Tauri or Pleiades' field flare stars a few have changing (may be cyclic) but definitely higher than average activity (SzécsényiNagy 1990) which may result in a recording of a flare spectrum in only some nights of observing.

\section{Conclusions}

Flare stars associated with open clusters or other kind of star systems have to be observed by FFMOSs. The most promising fields are that of the Pleiades (centred upon the star Eta Tauri) in the northern, and that of the Orion association in the southern hemisphere. Both are ideally placed for observatories equipped with the necessary wide-field telescopes and the new spectrographs. Recently developed systems (like Autofib) are user friendly enough and may contribute to the success of the program.

\section{Acknowledgments}

The author acknowledges the partial support of this research by the NSRF (grant OTKA-T 7595).

\section{References}

Haro G. \& Morgan W. W., 1953, ApJ, 117, 73

Mirzoyan L.V., 1990, in Star Clusters and Associations, B. A. Balázs \& G. Szécsényi-Nagy, eds, Roland Eötvös Univ. and the Konkoly Obs. of the Hung. Acad. of Sciences, Budapest, p.51

Schilbach E., Guibert J., Hirte S. \& Souchay J., 1994, these proceedings

Szécsényi-Nagy G., 1986, Publ. Astron. Dep. Eötvös Univ., No. 8, (Budapest), p.59\& p.101

Szécsényi-Nagy G., 1990, Ap\&SS, 170, 63

Tsvetkov M., Chukova M. \& Tsvetkova K., 1994, in Astronomy from Wide-Field Imaging, Proc. IAU Symp. 161, H. T. MacGillivray, ed., in press 


\section{Discussion}

MacConnell: Please compare and contrast the multi-object approach to the classical method of finding flare stars by taking many short, ultraviolet exposures in direct mode on one plate.

Szécsényi-Nagy: The traditional method of discovering flare stars and recording their flare-ups by taking 4 to 12 exposures on the same plate (moving the plate slightly between each exposure), in the $U, V$ or $B$ spectral band is only able to provide information on the celestial coordinates and approximate apparent brightnesses of the objects. Even photographic multi-colour photometry, made possible by the direct use of Schmidt cameras in extended sky fields, is unable to supply us with the spectral information neccessary for the accurate classification of flare stars and for the determination of their space velocities.

By using fibre-fed multi-object spectrographs, coupled with powerful Schmidt telescopes, we could collect spectra of these objects which contain much more detail than for spectra taken through objective prisms, and could also reach fainter sources. For these kinds of observations the MOSs offer an almost uniform dispersion in the whole optical band whereas prisms have heavily decreasing reciprocal linear dispersion from the ultraviolet to the infrared. In particular, we are absolutely unable to determine any emission features from objective prism spectra. Another aspect is the limiting magnitude. By using the classical photographic techniques, the sky background contributes its full photon flux to the spectra, whilst fibre-fed MOSs disperse the light collected from the direction of the observed star and its (seeing limited) surroundings. The result is a gain of 4-6 magnitudes depending on the actual dispersions. 